\title{
A Power Efficient Routing Protocol in Wireless Sensor Networks
}

\author{
Hyunsook Kim, Jungpil Ryu, and Kijun Han \\ Department of Computer Engineering, \\ Kyungpook National University, Korea \\ \{hskim, goldmunt\}@netopia.knu.ac.kr, kjhan@knu.ac.kr
}

\begin{abstract}
Saving energy consumption for a long network lifetime is one of the most important issues in design of wireless sensor networks because they usually consist of a large number of nodes with limited battery power. In this paper, we propose a balanced tree construction scheme called BATR (Balanced Aggregation Tree Routing), which uses near optimal minimal spanning tree for balancing the power consumptions over all nodes. Our main idea is that if energy consumed for transmission and reception can be nicely balanced over all nodes, an optimal data aggregation can be achieved in terms of network lifetime. Simulation results assure that the BATR can lengthen system lifetime as compared with the conventional data aggregation protocols.
\end{abstract}

\section{Introduction}

Recent advancements in wireless communication and highly progressed hardware technology have enabled the development of a wireless sensor with a low-cost processor, low-power, and light-weight. Each sensor collects useful information from the region in a variety of scenarios including military surveillance and landmine detection in harsh physical environments and monitoring [1]. Typically, sensor nodes collect audio, seismic, and other types of data and collaborate to perform a high-level task in a sensor web. Since wireless communications consume a significant amount of battery power, sensor nodes should be energy efficient in transmitting data. Energy efficient communication in wireless networks is attracting increasing attention in the literature.

Since sensor nodes might generate significant redundant data, data aggregation is usually used for wireless routing in sensor networks [2][3]. In each round of this datagathering application, all data from all nodes need to be collected and transmitted to the sink, where the end-user can access the data [9]. There are also different protocols proposed in literatures ([7][8]) so as to maximize the lifetime of the system under different circumstances. The simplest approach to routing in wireless sensor networks is direct transmission, in which each node transmits its own data directly to the sink.

In direct transmission, if the location of sink is far from sensor field, each sensor node dissipates a large amount of energy.

\footnotetext{
Correspondent author.
} 
LEACH(Low-Energy Adaptive Clustering Hierarchy)[9] is proposed in order to efficiently do data gathering and aggregation. $\mathrm{LEACH}$ is a clustering-based protocol that utilizes randomized rotation of the cluster-heads to evenly distribute the energy load among the sensor nodes in the network. It assumes that the sink is fixed and located far from the sensors, and all nodes in the network are homogeneous and energy-constrained. But, LEACH is impractical, since it is directly transmission method. Thus, It is desirable to multi-hop transmit in wireless sensor network In [10], the authors propose a new minimum spanning tree-based protocol, called PEDAP (Power Efficient Data Gathering and Aggregation Protocol) and its power-aware version (PEDAP-PA). In PEDAP, the locations of all nodes are known by sink a priori, and path information of network is computed by sink. PEDAP is based on minimum spanning tree routing scheme as well. These previous research and concurrent works on protocol and algorithm designed in sensor networks are summarized in [1][4][5].

In this paper, we propose a balanced-tree based routing protocol called BATR (Balanced Aggregation Tree Routing) to achieve an optimal data aggregating in terms of the network lifetime by balancing power consumption per node. In our scheme, each intermediate node keeps the number of children nodes and it transmits the data to the nearest neighbor node. Our scheme may minimize the energy consumption in each node, thus prolong the lifetime of the system regardless of location of the sink is outside or inside the sensor field.

The rest of the paper is organized as follows. The BATR protocol is described in detail in Section 2. Next, in Section 3, we present our simulation results. Finally, we conclude the paper and present future research directions in Section 4.

\section{Balanced Aggregation Tree Routing Protocol}

\subsection{System Model}

Data aggregation, which is the combination of data from different multiple sources, is needed for saving energy by reducing the number of messages in the network. In general, data gathering requires some energy at intermediate nodes. To investigate power consumption in wireless sensor network, we use a radio model as described in [12], which is the first free space channel model where the power loss $d^{2}$ is used. First, we formally model the network as a graph $\mathrm{G}=\{V, E\}$ where $V$ is the set of nodes $\{0, \ldots \ldots, n\}$ in the network and $E$ is set of edges between nodes. We assume that combining $n$ packets of size $k$ results in one packet of size $k$ instead of size $n k$ [10]. We define the edges by $\forall x$ and $\forall y \in V, \exists(x, y) \in E \Leftrightarrow \operatorname{dis}(x, y) \leq d$, where $d$ is the transmission range of sensor nodes in the network and dist $(x, y)$ is the Euclidean distance between nodes $x$ and $y$ which are said to be neighbors of each other. The set of neighbors of a node $\mathrm{X}$ is denoted as neighbors $(\mathrm{X})$. For a tree $\mathrm{T}$ constructed for aggregation tree from graph $\mathrm{G}$, we call a set $X \subseteq V$, a group iff $X=\{Y\} \mathrm{Y} Z$ where $\mathrm{Y}$ is the parent of nodes of $Z$ in the tree $T$ [11].

To transmit a k-bit packet over a distance $d$, the radio expends

$$
E_{T x}(k, d)=k E_{\text {elec }}+k d^{2} \varepsilon_{f s}
$$

to receive this message, the radio expends 


$$
E_{R x}(k)=k E_{\text {elec }}
$$

In these radio parameters, $E_{\text {elec }}$ means a radio dissipates to run the transmitter or reciever circuitary and $\varepsilon_{f s}$ for the transmitter amplifier separately.

As seen above, since the energy cost consists of two components, one for transmitting or receiving a packet in the radio electronics and the other depending on the distance in transmissions, the routing protocols must minimize the number of data receptions and while minimizing the transmit distance for a specific node. In order to maximize the network lifetime, the total energy should be minimized in a round of communication, while balancing the energy consumption among the nodes. An ideal aggregation tree can be thought as a balanced tree with a minimum number of edges, equivalent children nodes in order to maximize the network lifetime. For this, our protocol is designed to find an optimal path based on a balanced tree that has almost the same number of children nodes below itself. Here, the child node means the node that could exist in any neighborhood.

To describe our protocol, we assume that the system consists of $n$ sensors distributed randomly in the environment that is being monitored. We assume that sink knows the locations of all nodes priori either by manually entering coordinates or by using the GPS. Each node periodically senses its nearby environment and would like to send its data to a sink. The sensor nodes, including the sink in the network have equal transmission range (say $d$ ). The nodes aggregate or fuse the data they receive from the others with their own data, and produce only one packet regardless of how many packets they receive. In addition, it is assumed that all nodes generate equal amount of data, and there is no mobility in the network[10].

\subsection{Detailed Procedure}

In our protocol, the routing information is computed using a MST(Minimum Spanning Tree) algorithm. Given a topology graph $G(V, E)$, it begins with the root node and starts iteratively establishing parent-child relationship with other nodes. Sink functions as a root node in MST.

(1) Initially, we start the path construction from sink as a root.

(2) After that, we select the nearest nodes among neighbors as many as the number of decided children nodes from a vertex in the tree to a vertex not in the tree, and add that edge to the tree. In our case, data will be send through that edges just included in the tree from each node. In our protocol, we balance the number of children nodes depending on the density of network to achieve a long lifetime. Here, the number of children nodes, denoted by $\mu$, is given as an operational parameter whose value can be calculated by

$$
\mu(R)=\alpha \frac{N \pi R^{2}}{A}
$$

where $N$ is the number of scattered sensor nodes in region $A$, and $R$ can be either the range of a particular sensor or the radio transmission range (idealized with circular propagation) [13]. Basically, $\mu(R)$ gives the number of nodes within 
the transmission radius of each node in region $A . \alpha$ is a variable which depends on the position of sink. If the number of children nodes is $a$, energy consumption of intermediate node for data aggregation is

$$
E_{\text {intermediate }}(k, a, d)=k E_{\text {elec }}+k d^{2} \varepsilon_{f_{s}}+a\left(k E_{\text {elec }}+k E_{D A}\right)
$$

So, the total energy expended by the network is given by the sum of energies used by each node $\left(E_{\text {Total }}=\sum_{i \in N} E_{i}\right)$.

(3) When neighbor node is found over the limit of search, the node is labeled as a leaf node. We set the search limit at 15 for prevention of delay.

(4) We repeat this procedure until all available nodes are added to the tree.

(5) After some certain number of rounds, the sink recomputes the routing information considering the condition of nodes and resetting the number of children nodes. After each computation, the sink sends each node the required routing information for that node such as the node's parent in the tree in order to reach to the sink and the number of child nodes, etc.

Fig. 1 shows the process of tree construction and the result of routing paths for a sample network when children nodes are under two. A dotted line indicates the path which is node's aggregation information including death node. And solid line shows the resulting routing paths for a sample network.

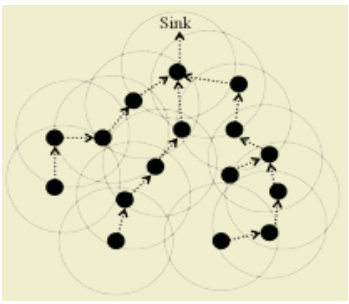

(a) Aggregation of node's information

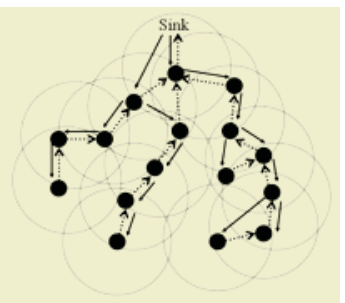

(b) Result of routing paths

Fig. 1. An example for construction of a balanced tree

\section{Simulation Results}

We use the simulation programs written in $\mathrm{C}++$ program language to evaluate the performance of our scheme. Our scheme is compared with direct transmission, LEACH, and non-balanced routing scheme which uses Prim's spanning tree algorithm. The network dimensions for our experiments is $100 \times 100$ and 100 nodes are randomly generated within the region. We run the simulations with different position of sink: to the center of the field and to the place away from the region.

Table 1. gives the parameters for the energy dissipation of the radio in order to run the transmitter or receiver circuitry. 
Table 1. Parameters for simulation

\begin{tabular}{l|c|l}
\hline \hline Description & Parameter & Value \\
\hline \hline Radio electronics & $E_{\text {elec }}$ & $50 \mathrm{~nJ} / \mathrm{bit}$ \\
\hline Transmit amplifier & $\varepsilon_{f s}$ & $100 \mathrm{pJ} / \mathrm{bit} / \mathrm{m}^{2}$ \\
\hline Aggregation cost & $E_{D A}$ & $5 \mathrm{~nJ} / \mathrm{bit} / \mathrm{signal}$ \\
\hline Data size & $D_{\text {size }}$ & $1000 \mathrm{bits}$ \\
\hline
\end{tabular}

Fig. 2 shows the number of rounding of death nodes for networks where the sink is located in the center of the field. In general, the direct transmission can offer a satisfactory performance when the transmission distance between the node and the sink is not so far. However, BATR shows a similar performance to the direct transmission after the number of dead nodes reaches $50 \%$. We can predict this result from Eq. (4) since there is no child node when more than $50 \%$ of nodes are dead. Taken as a whole, BATR improves the lifetime about 1.5 times compared with non-balanced tree protocol.

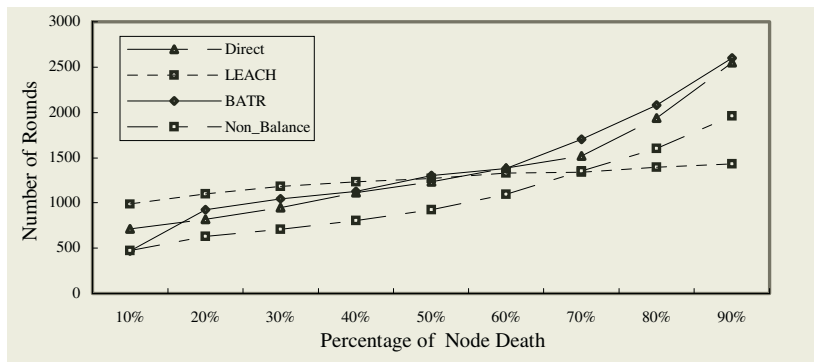

Fig. 2. Lifetime for a $100 \times 100$ network where the sink is at the center of the field

Fig. 3 indicates that BATR is the best power saving system when sink is far away from the field. As seen in Fig. 4, the lifetime obtained with direct transmission is very short since if the sink is far away from nodes, the nodes will consume more energy for data transmission and so die quickly. In case of non-balanced tree, distant nodes should send data directly after a certain time since nodes around the sink die first. On the other hand, dead nodes will be distributed uniformly over the network in BATR since the distances among nodes are not so far.

Fig. 4(a) shows that the lifetime as the number of children nodes is varied. It is worth to note that our scheme also performs well when the sink is outside the field. As shown from Eq. (1), energy consumption of each node is proportional to the square of distance between nodes. When the number of children nodes is incremented, the depth of tree is decreased but the distance between nodes becomes large. So, each node will consume more energy at this time. In our experiments, we can see that the longest lifetime can be achieved when there are two children nodes in case that sink is located far away from the field. Further, as we increase the number of children nodes, the network lifetime becomes shortened. 


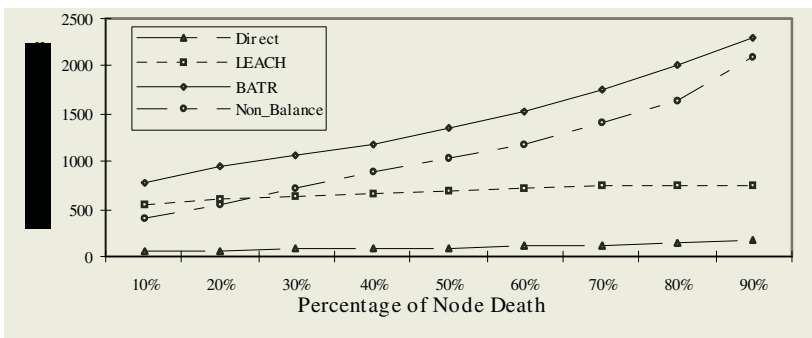

Fig. 3. Lifetime for a $100 \times 100$ network where the sink is outside of the network

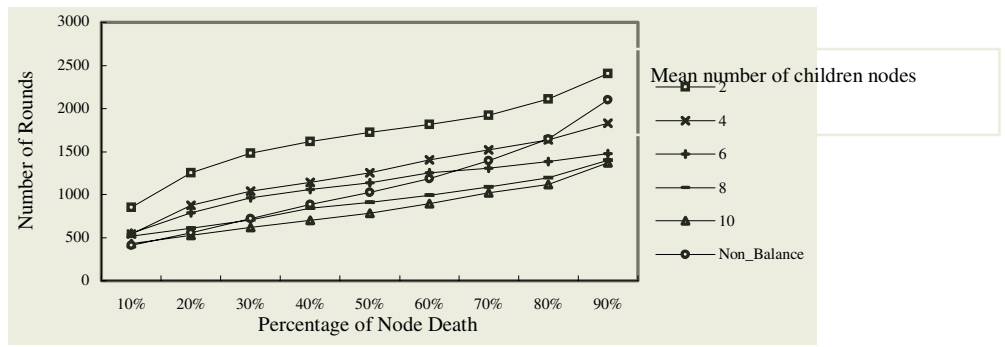

(a) When the sink is located at the center of the field

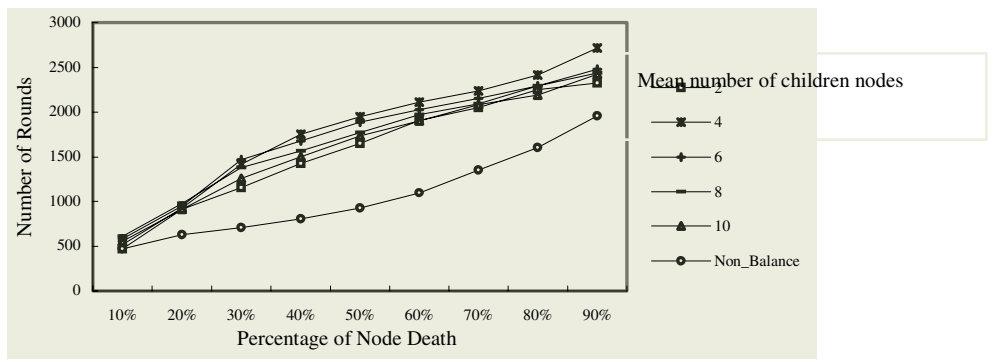

(b) When the sink is located outside of the field

Fig. 4. Lifetime versus the number of children nodes

Fig. 4(b) represents a lifetime versus the number of children nodes when sink is located at the center of the field. We can observe almost the same lifetime regardless of the average number of children nodes. This is because the number of children nodes does not affect the energy consumption so much while the distance between nodes is the most dominant factor to the energy consumption.

To evaluate distribution characteristics of energy consumption in the network, we measure standard deviation of loads over all sensor nodes after each round. A large variance in load signifies that load is not uniformly distributed among the nodes. Fig. 5 demonstrates that our approach outperforms the non-balanced tree protocols. 


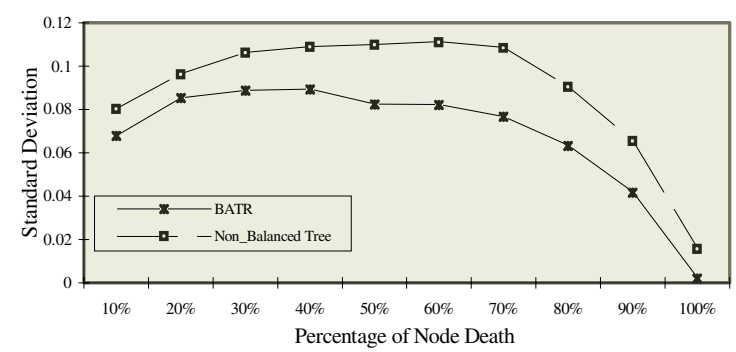

(a) When the sink is located outside of the field

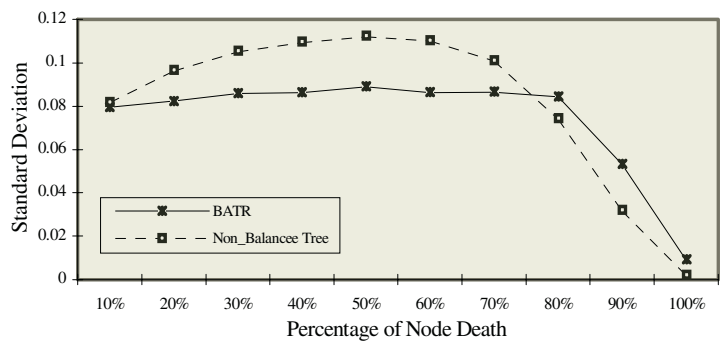

(b) When the sink is located in the center of the field

Fig. 5. Standard deviation of loads as the number of dead sensors is increased

As previously described, the energy cost consists of two components, one is the number of children nodes and the other is transmission distance. Therefore, the routing protocols must minimize the number of data receptions and reduce the distance of transmission between specific nodes. In BATR, a node with large transmission distance becomes a leaf node on a tree. So, it can save energy because it does not need aggregation and receiving. In case of intermediate nodes, the deviation of energy consumption can be reduced by balancing the number of children nodes.

\section{Conclusion and Future Works}

Distributing the load evenly to the nodes has a great impact on system lifetime. In this paper, we propose a power efficient routing protocol based on a balanced tree. Our main idea is minimizing the total energy of the system by balancing of energy consumption among the nodes. We show through simulations that our scheme performs near optimal regardless of the position of the sink and outperforms the conventional approaches such as LEACH and non-balanced tree protocols.

Our future work involves several refinements and extensions of this work, as well as establishment of analytical models to get more comprehensive underlying performance limit. 


\section{Acknowledgement}

This research is supported by Program for the Training of Graduate Students for Regional Innovation.

\section{References}

1. I. Akyildiz, W. Su, Y. Sankarasubramaniam, and E. Cayirci, "A Survey on Sensor Networks," IEEE Communications Magazine, Vol. 40, No. 8, pp. 102-116, August 2002.

2. J.Heidemann, F.Silva, C.Intanagonwiwat, R. Govindan, D.Estrin, and D. Ganesn, "Building Efficient Wireless Sensor Networks with Low-Level Naming", $18^{\text {th }}$ ACM Symposium on Operating Systems Principles, October 21-24,2001

3. C. Intanagonwiwat, R.Govindan and D.Estrin, "Directed Diffusion: A Scalable and Robust Communication Paradigm for Sensor Networks," ACM/IEEE International Conference on Mobile Computing and Networks(MobiCom2000), August 2000, Boston, Massachusetts

4. J.-H. Chang and L.Tassiulas, "Energy conserving routing in wireless ad-hoc networks," in IEEE INFOCOM 2000, March 2000, pp 22-31.

5. J. Chang and L. Tassiulas, "Maximum lifetime routing in wireless sensor networks," in Proceedings of Advanced Telecommunications and Information Distribution Research Program, College Park, MD, 2000

6. M. Bhardwaj, A. Chandrakasan, and T. Garnett, "Upper bounds on the lifetime of sensor networks," in IEEE International Conference on Communications, 2001, pp. 785-790.

7. W. Heinzelman, J. Kulik, and H. Balakrishnan, "Adaptive protocols for information dissemination in wireless sensor networks," in MOBICOM, 1999, pp. 174-185.

8. K. Kalpakis, K. Dasgupta, and P. Namjoshi, "Maximum lifetime data gathering and aggregation in wireless sensor networks," in Proceedings of the 2002 IEEE International Conference on Networking (ICN'02), August 2002, pp. 685-696.

9. W. R. Heinzelman, A. Chandrakasan, and H. Balakrishnan, "Energy efficient communication protocol for wireless microsensor networks," in 33rd Annual Hawaii International Conference on System Sciences, , 2000, pp. 3005 - 3014.

10. Hüseyin Özgür Tan and Îbrahim Körpeogle , "Power Efficient Data Gathering and Aggregation in Wireless Sensor Networks," in SIGMOD Record, Vol, 32, No. 4. Dec 2003,

11. V. Annamalai, SKS and Gupta L. Schwiebert, "On Tree-based convergecasting in wireless sensor networks," IEEE Wireless Communications and Networking Conference $(W C N C)$,pp 1942-1947, March 2003

12. W. R. Heinzelman, A. Chandrakasan, and H. Balakrishnan, "An Application-Specific Protocol Architecture for Wireless Microsensor Networks," IEEE Transactions on Wireless Communications, Vol. 1, No. 4, Oct 2002

13. Bulusu et al., "Scalable Coordination for Wireless Sensor Networks : Self-Configuring Localization Systems," ISCTA 2001, Ambleside, U.K., July 2001 